\title{
Investigation of Polyurethane Electrospinning Process Efficiency
}

\author{
Dusan Kimmer ${ }^{\mathrm{a}}$, Martin Zatloukal ${ }^{\mathrm{b}}$, David Petras ${ }^{\mathrm{a}}$, Ivo Vincent ${ }^{\mathrm{a}}$ and \\ Petr Slobodian ${ }^{\mathrm{b}}$ \\ ${ }^{a}$ SPUR a.s., T. Bati 299, 76422 Zlin, Czech Republic \\ ${ }^{b}$ Polymer centre, Faculty of Technology,Tomas Bata University in Zlin, TGM 275, 76272 Zlin, \\ Czech Republic
}

\begin{abstract}
The electrospinning process efficiency of different PUs has been investigated. Specific attention has been paid to understand the role of PU soft segments and synthesis type on the stability of the PU solution and electrospinning process as well as on the quality/property changes of the produced nanofibres. PU samples before and after the process were analyzed rheologicaly and relaxation spectra were determined for all of them from frequency dependent loss and storage moduli measurements. It has been found that rheological analysis of PU, which is used for electrospinning process, can be useful tool from electrospinning process efficiency and optimization point of view. Nanolayers homogeneity during several hours of manufacture in optimized electrospinning process is proved by selected properties from aerosol filtration.
\end{abstract}

Keywords: Nanofiber, Electrospinning, Polyurethanes, Rheological analysis, Relaxation spectra, Nanolayers homogeneity, Process stability, Continues manufacture.

PACS: $62.23 . \mathrm{St}$, 68.37.Hk, 81.16.Be, 81.16.Rf, 82.35.Lr, 82.45.Yz, 87.85.Uv

\section{INTRODUCTION}

Electrospinning is technology, which can be used for production of nanofibers [1-14]. One of the main disadvantage of the nanofiber based structures is their weakness [9-12]. This undesirable property can be improved by the use of suitable polymer such as polyurethane (PU) which has high elasticity. PUs are segmented polyadducts build-up from soft (polyol) and hard (created from diisocyanate and chain extender) segments. In this work, the process stability/efficiency with respect to final nanofiber product quality was investigated.

\section{EXPERIMENTAL}

Nanofibers were prepared from PU solutions in dimethylformamide (DMF) by using commercially available Nanospider equipment (http://www.elmarco.com) employing one rotational electrode with needles. Experimental conditions were as follow: Relative humidity $R H \sim 29 \%$, temperature $T \sim 25^{\circ} \mathrm{C}$, electric voltage $U=75 \mathrm{kV}$, distance between rotating and static electrode $L=18 \mathrm{~cm}$, electrode spin $R=7 \mathrm{r} / \mathrm{min}$., speed of antistatic polypropylene nonwoven fabric collecting nanofibres $S=0.16 \mathrm{~m} / \mathrm{min}$. PU solutions in DMF were synthesized from 4,4' - methylenebis (phenyl isocyanate) (MDI), polymer diol $\left(M_{\mathrm{n}} \sim 2.10^{3}\right)$ and 
1,4-butanediol (BD) in molar ratio 6:1:5 (9:1:8 eventually) at $90^{\circ} \mathrm{C}$ for 5 hours. The following polymer diols, which introduces different type of soft segments in PU, were used: polytetramethyleneoxide (PTMO), poly(1,4-butanediol)-co-(adipic acid) (PBA), poly(3-methyl-1,5-pentanediol)-alt-(adipic, isophtalic acid) (PAIM), poly(3-methyl-1,5-pentanediol)-co-(adipic acid) (PAM) and copolymer based on diethyl ester carbonic acid with 1,6-hexanediol and 3-methyl-1,5-pentanediol (PCHM). All PU solution viscosities $(\eta)$ were adjusted to be equal to $1.5 \mathrm{~Pa} . \mathrm{s}$ (which corresponds to PU 10.5-15.8 wt.\% concentration in DMF) having conductivity $\chi \sim 150 \mu \mathrm{S} / \mathrm{cm}$ (adjusted by tetraethylamonium bromide). PUs were synthesized firstly in "One shot" (Os) where all reactants were added in one step and secondly "Per partes" (Pp) where the prepolymer was synthesized from MDI and polymer diol (molar ratio 2:1) firstly and in the second step, all extender (BD) was added and remaining MDI quantity was added after 1 hour of polyaddition reaction.

The PU solutions before and after the process were analyzed rheologicaly by using rotational reometer ARES 2000 whereas nanofiber structure analyses have been done by using Scan Electron Microscope JSM 840 (JEOL). Penetration of aerosol was measured by means of filter measuring system LORENZ adjusted for EN 143.

\section{RESULTS AND DISCUSSION}

Structural stability/changes of the PU solution during the electrospinning process and product manufacture quality are discussed here in more details. PU samples before the process, after several hours of the process and PU samples which nanofibres are created from where analyzed rheologicaly and relaxation spectra were determined for all of them from frequency dependent loss and storage moduli measurements. The results are depicted in Figures 1-8.

\section{Approximation - Synthesis Optimalization, Stationary Electrospinning Process}

Set of PU samples, containing PBA soft segment, with varying molar ratio MDI:PBA:BD from 2:1:1 to $6: 1: 5$, were synthesized and fibrillated in electric field. The best nanofibres quality was reached from molar ratio 6:1:5 and that is why the PU based on other polymer diols were synthesized with the same molar ratio.

In Figure 1 it can be seen that relaxation spectrum for PTMO based PUs (prepared by Os synthesis) differ significantly if the PU samples taken before and after 3 hours of the process are compared. In this case, the quality of the produced nanofibers was change in the time as well. On the other hand, PTMO based PU (prepared by Pp synthesis) has shown no big differences in the quality of the produced nanofibers during 3 hours process which is reflected by no big changes in the relaxation spectra for original and processed PU samples (see Figure 2). 


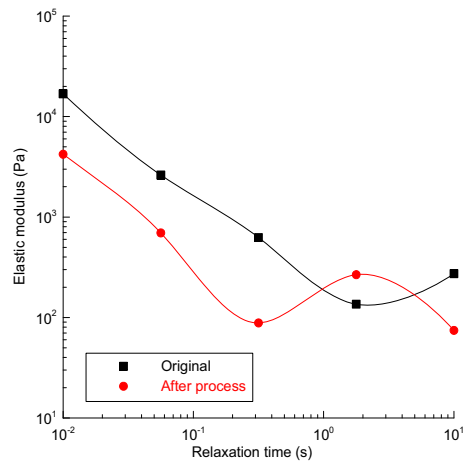

FIGURE 1. Relaxation spectrum of PU based on PTMO, Os way of synthesis.

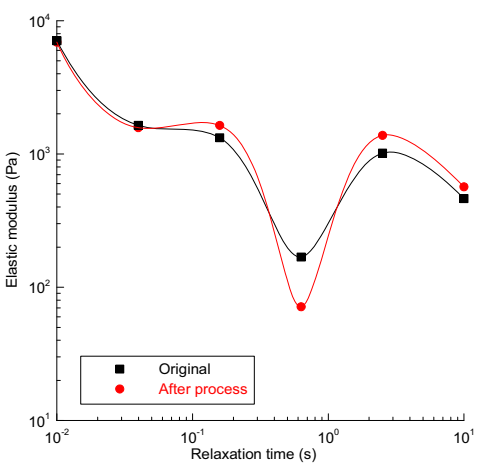

FIGURE 2. Relaxation spectrum of PU based on PTMO, Pp way of synthesis.

\section{Approximation - Influence of Soft Segments Chemical Composition}

In Figures 3-5, the relaxation spectra for original and processed PU samples prepared by Pp syntheses, having different types of the soft segments, are depicted. It has been revealed that PUs with PAIM and PCHM soft segments were the best from the time dependent stability and nanofibers quality point of view. It has been found that it correlates very well with low variation in the relaxation spectra within the processing time as visible in Figures 4-5.

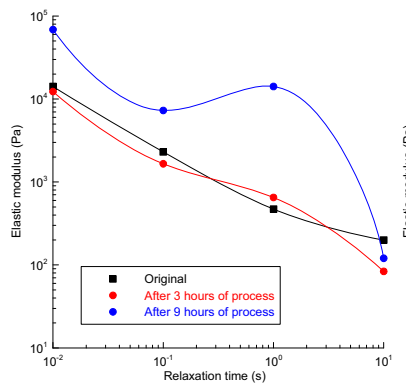

FIGURE 3. Relaxation spectrum of PU based on PAM, Pp way of synthesis.

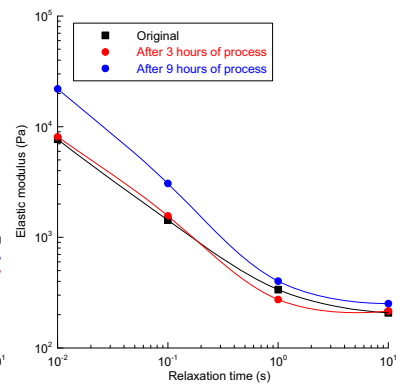

FIGURE 4. Relaxation spectrum of PU based on PCHM, Pp way of synthesis.

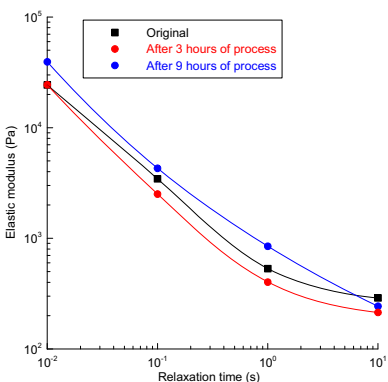

FIGURE 5. Relaxation spectrum of PU based on PAIM, Pp way of synthesis. 


\section{Approximation - Rheological Properties Comparison of PU in Treated Solution and Nanofibres}

Relaxation spectra comparison of PUs from spinning bath and fibrillated PU (samples were prepared by dissolving of nanofibres and subsequent evaporation of DMF) prove the best workability for PU with PAIM soft segment. Such a PU can be used in long term electrospinning process and even for close-cycle technology. Spectra of PUs from nanofibres show constant values of elastic modulus in wide interval of relaxation times, the formation of double linkage conjugated system in PU chains (connected also with browning of PU) being the reason.

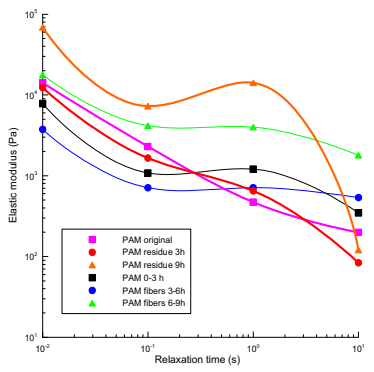

FIGURE 6. Relaxation spectra of PUs based on PAM, comparison of PUs from fibrillated solution and nanofibres.

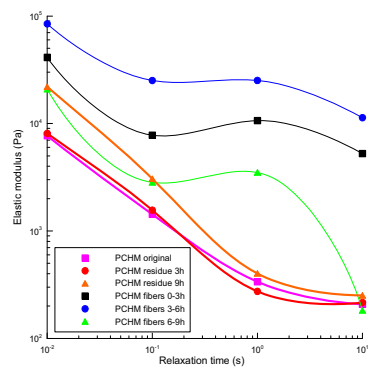

FIGURE 7. Relaxation spectra of PUs based on PCHM, comparison of PUs from fibrillated solution and nanofibres.

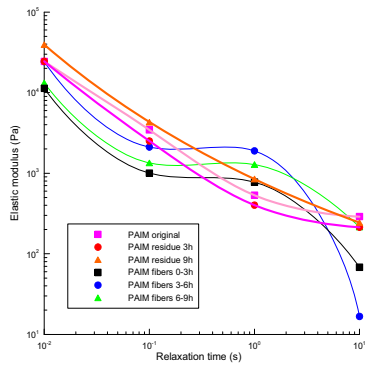

FIGURE 8. Relaxation spectra of PUs based on PAIM, comparison of PUs from fibrillated solution and nanofibres.

Figure 9 illustrates the changes in quality of nanofibers which were detected by rheology analysis. Process efficiency (expressed as dependence of area mass on concentration of PU in solution) decrease with process duration in case of bigger variation in relaxation spectra. 


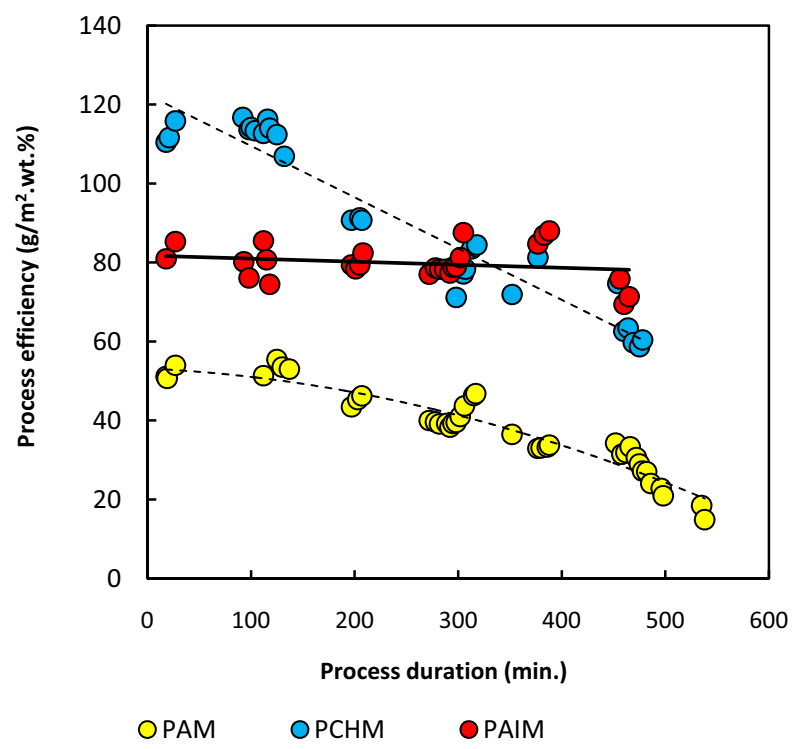

FIGURE 9. Changes of area mass with process duration, influence of soft segments chemical composition.

\section{Approximation - Product Manufacture Quality and Properties Changes During Spinning Process}

One of the easiest but very large application of layers based on nanofibres is filtration. Uniformity of nanofibres deposition during several hours of process was traced by measurement of area mass (Figure 10), capture of aerosol particles under EN 143 characterized by pressure resistance (Figure 11), aerosol penetration (Table 1) and by means of SEM (Figures 12-14).

Relative humidity at spinning process and molar ratios of synthesized PUs are alternated variables at production of nanolayers compared on Figure 10. Process efficiency characterized by area mass increases with higher content of hard segments in PU structure. Lower efficiency in case of lesser value of relative humidity proves the coinitiating effect of water at electrospinning process. 


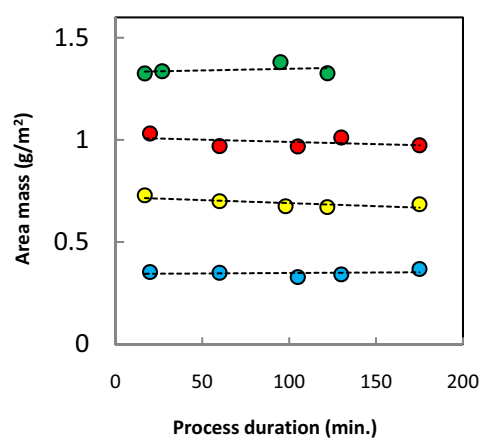

O PAIM 615, $\mathrm{RH}=17 \%$ O PAIM 918, $\mathrm{RH}=29 \%$

O PAIM $615, \mathrm{RH}=29 \%$ O PAIM $615, \mathrm{RH}=37 \%$

FIGURE 10. Process stability, coinitiating effect of water, molar ratio influence of synthesized PU.

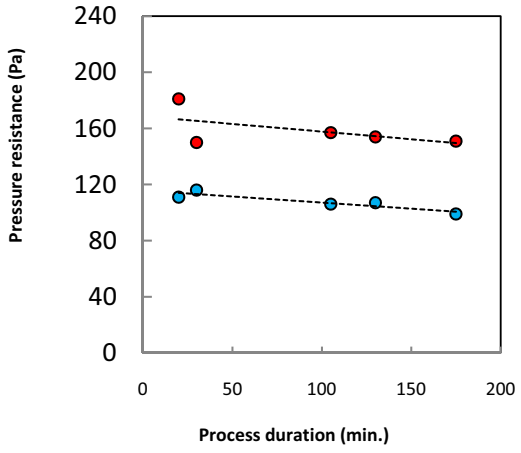

- PAIM 615 O PAIM 918

FIGURE 11. Uniformity of nanolayers filtration properties.

Aerosol penetration measurements (Table 1) were done at flow $5.6 \mathrm{~cm} / \mathrm{s}$ on various places of manufactured band from nanolayers based on PU 918 with area mass $\sim 2.9 \mathrm{~g} / \mathrm{m}^{2}$ prepared between 30 and $120 \mathrm{~min}$ of electrospinning process.

TABLE 1. Reproducibility of aerosol penetration.

\begin{tabular}{c|rrrrrrr}
$\begin{array}{c}\text { Pressure drop } \\
(\mathbf{P a})\end{array}$ & 659 & 599 & 521 & 617 & 544 & 659 & 620 \\
\hline $\begin{array}{c}\text { Aerosol } \\
\text { penetration } \\
\mathbf{( \% )}\end{array}$ & 0.00054 & 0.00110 & 0.00949 & 0.00832 & 0.00196 & 0.00092 & 0.00101
\end{tabular}

\begin{tabular}{c|rrrrrrr}
$\begin{array}{c}\text { Pressure drop } \\
(\mathbf{P a})\end{array}$ & 647 & 598 & 624 & 561 & 581 & 653 & 599 \\
\hline $\begin{array}{c}\text { Aerosol } \\
\text { penetration } \\
(\%)\end{array}$ & 0.00036 & 0.00093 & 0.00074 & 0.00108 & 0.00082 & 0.00054 & 0.00104 \\
\hline$\%$ & & & & & & &
\end{tabular}




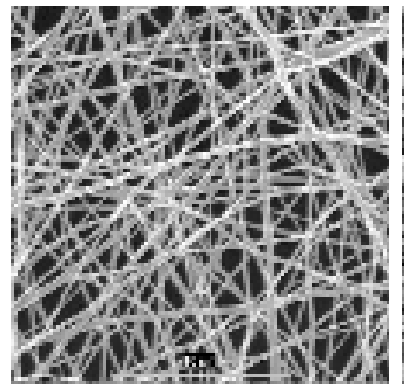

FIGURE 12. Nanofibres made of PU 615 based on PAIM after 20 min. of electrospinning process.

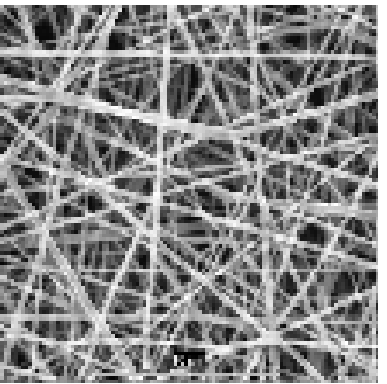

FIGURE 13. Nanofibres made of PU 615 based on PAIM after $175 \mathrm{~min}$. of electrospinning process.

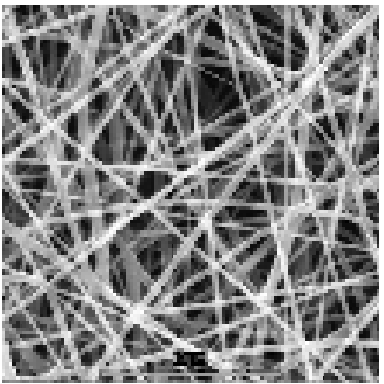

FIGURE 14. Nanofibres made of PU 615 based on PAIM after 367 min. of electrospinning process.

\section{CONCLUSIONS}

The first technological request for potential application of layers from nanofibres is production of material with constant properties during the whole continues manufacture. It has been revealed experimentally that an application of PU based on PAIM soft segment satisfies this important technological request. It has also been found that rheological analysis of PU, treated in electrospinning process, can be useful tool from electrospinning process efficiency and optimization point of view.

\section{REFERENCES}

1. J. Doshi and J. D. Reneker, Journal of Electrostatics 35, 151-160 (1995).

2. H. Zhuo, J. Hu, S. Chen and L. Yeung, J. Appl. Polym. Sci. 109, 406-411 (2008).

3. J. A. Matthews, G. E. Wnek, D. G. Simpson and G. L. Bowlin, Biomacromolecules 3, 232- 238 (2002).

4. M. M. Demir, I. Yilgor, E. Yilgor and B. Erman, Polymer 43, 3303- 3309 (2002).

5. C. L. Casper, J. S. Stephens, N. G. Tassi, D. B. Chase and J. F. Rabolt, Macromolecules 37, 573-578 (2004).

6. Y. Z. Zhang, C. T. Lim, S. Ramakrishna and Z. M. Huang, J. Matter Sci: Mater. Med. 16, 933- 946 (2005).

7. R. Inai, M. Kotaki and S. Ramakrishna, Nanotechnology 16, 208-213 (2005).

8. Z. M. Huang, Y. Z. Zhang, M. Kotaki and S. Ramakrishna, Compos. Sci. Technol. 63, 2223-2253 (2003).

9. J. M. Lyons, Melt-Electrospinning of Thermoplastic Polymers: An Experimental and Theoretical Analysis Philadelphia: Drexel University, 2004.

10. A. Pedicini and R. J. Farris, Polymer 44, 6857-6862 (2003).

11. J. P. Anderson, K. McGrath and D. Kaplan, In Protein-Based Materials Basel: Birkhauser, 1997.

12. J. Ayutsede, M. Gandhi, S. Sukigara, H. H. Ye, C. M. Hsu, Y. Gogotsi and F. Ko, Biomacromolecules 7, 208-214 (2006).

13. D. Kimmer, M. Zatloukal, M. Tomášek, D. Petráš, T. Dudák and Z. Dudák, “Optimized nanofiber production". Patent filed at Czech patent office (UPV CR). No. PV 2008-849.

14. D. Kimmer, P. Slobodian, D. Petras, M. Zatloukal, R. Olejnik and P. Saha, J. Appl. Polym. Sci. 111, 2711-2714 (2009). 
Copyright of AIP Conference Proceedings is the property of American Institute of Physics and its content may not be copied or emailed to multiple sites or posted to a listserv without the copyright holder's express written permission. However, users may print, download, or email articles for individual use. 